\title{
Hepatitis B Reactivation: A Potentially Serious Complication of Chemotherapy for Solid Tumors
}

\author{
Sandra Algaze ${ }^{2}$, John Donovan² and Irene Kang1* \\ ${ }^{1}$ Keck School of Medicine Division of Medical Oncology, University of Southern California, Los Angeles CA \\ ${ }^{2}$ Keck School of Medicine Division of Gastrointestinal and Liver Diseases, University of Southern California Los Angeles CA
}

*Corresponding author: Irene Kang, university of Southern California, Keck School of Medicine, 1441 Eastlake Ave. Los Angeles, USA.

Received Date: January 02, 2019

Published Date: January 22, 2020

Abstract

We present a case of hepatitis B reactivation in a patient receiving adjuvant chemotherapy for early breast cancer. This is a 49-year-old post-menopausal, Chinese female with history of chronic hepatitis B with inactive carrier state, and right invasive ductal carcinoma staged as CT2N1M0, pT2N1aM0, anatomic stage IIB, prognostic stage IIA, estrogen and progesterone receptor positive, and human epidermal growth factor 2 negative. The patient underwent bilateral mastectomy with implant reconstruction and right axillary lymph node dissection followed by adjuvant chemotherapy planned as dose dense doxorubicin and cyclophosphamide (ddAC), followed by dose dense paclitaxel (ddT). Two weeks after her first cycle of dose dense paclitaxel, she developed grade III HBV reactivation with hepatitis. This report highlights HBV reactivation as a potential complication of chemotherapy for solid tumors, and reviews recommended HBV screening, prevention and management of HBV reactivation in this patient population.

Keywords: Hepatitis b virus; Reactivation; Hepatitis; Solid tumors; Cancer; Chemotherapy; Antiviral; Prophylaxis; Screening; Breast cancer

Abbreviations : AALD - American Association for the Study of Liver Disease; AGA - American Gastroenterological Association Institute; ALT - alanine transferase; ASCO - American Society of Clinical Oncology; AST - aspartate aminotransferase; CDC - US Centers for Disease Control and Prevention; ddAC - Dose dense Doxorubicin Cyclophosphamide; ddT - Dose Dense Paclitaxel; ER - Estrogen Receptor; ESMO - European Society for Medical Oncology; FISH - Fluorescence in situ hybridization; HBV - Hepatitis B Virus; HBcAb - Hepatitis B Core antibody; HBeAg - Hepatitis B e antigen; HBsAb - Hepatitis B surface antibody; HBsAg - Hepatitis B surface antigen; HBV DNA - Hepatitis B Virus Deoxyribonucleic Acid; HCC hepatocellular carcinoma; HER2 - human epidermal growth factor receptor 2; INR - International normalized ratio; NCCN - National Comprehensive Cancer Network; PR-Progesterone Receptor; TACE-transarterial chemoembolization; TNF- $\alpha$ - Tumor Necrosis Factor-alpha

\section{Introduction}

Chemotherapy is an important component in the management of various solid tumors. Patients with history of hepatitis B virus (HBV) or chronic HBV receiving chemotherapy for solid tumors are at risk for HBV reactivation. HBV reactivation may be associated with significant morbidity, mortality, and chemotherapy interruption. Preemptive antiviral therapy is effective in preventing HBV reactivation and associated complications in patients with chronic HBV. We present a case of HBV reactivation secondary to adjuvant immunosuppressive chemotherapy for breast cancer to illustrate the importance of appropriate screening prior to the initiation of chemotherapy to allow for HBV reactivation prevention, monitoring, and management.

\section{Case Report}

A 49-year-old post-menopausal, Chinese female presented with a palpable breast mass and was found to have a right invasive ductal carcinoma, that was positive for estrogen receptor (ER 95\%) and progesterone receptor (PR 50\%) and negative for human epidermal growth factor receptor 2 (HER2) by Fluorescence in situ hybridization (FISH). Her breast cancer was staged as cT2N1M0, pT2N1aM0, anatomic stage IIB, prognostic stage IIA with modified Scarff-Bloom-Richardson Score 6/9 and Nottingham Grade 2. She elected to undergo bilateral mastectomy with implant reconstruction and right axillary lymph node dissection with 3 out 18 positive lymph nodes and negative margins. The patient was subsequently started on adjuvant chemotherapy with dose dense 
doxorubicin $60 \mathrm{mg} / \mathrm{m}^{2}$ and cyclophosphamide $600 \mathrm{mg} / \mathrm{m}^{2}$ (ddAC), followed by dose dense paclitaxel $175 \mathrm{mg} / \mathrm{m}^{2}$ (ddT). The four cycles of anthracycline-containing chemotherapy were initially complicated by mild nausea, diarrhea, mucositis, mild anemia and grade 4 neutropenia without fever. Likely related to her prior anthracycline exposure, but two weeks during treatment with dose dense paclitaxel, the patient developed grade III hepatitis.

Her exam was benign and laboratory work-up revealed elevated transaminases (aspartate aminotransferase (AST): 2,059 U/l, ALT: 2,388 U/l), hyperbilirubinemia (total bilirubin: $1.8 \mathrm{mg} /$ $\mathrm{dl}$, direct bilirubin: $1.6 \mathrm{mg} / \mathrm{dl}$ ), without coagulopathy (international normalized ratio [INR]:1.1). Abdominal ultrasound with doppler was negative for acute pathology, and liver and gallbladder were unremarkable. Further assessment revealed positive hepatitis B surface antigen (HBsAg), hepatitis B core antibody (HBcAb), hepatitis B surface antibody (Anti-HBs), hepatitis B e Ab (HBeAb), and quantitative HBV DNA of 6,990,000 IU/ml. At this point, the patient noted a history of chronic hepatitis B, inactive carrier state without history of liver disease or treatment. Chemotherapy was held and she was started on tenofovir 300mg daily. A trend of her liver function tests is demonstrated in Figure 1. After one month of antiviral therapy, HBV DNA reduced to $73.9 \mathrm{IU} / \mathrm{mL}$. Transaminases and bilirubin normalized after two months of therapy. Although recommended to resume adjuvant chemotherapy, the patient ultimately deferred any further chemotherapy due to concern for further hepatic injury and was started on adjuvant endocrine therapy (Figure 1).

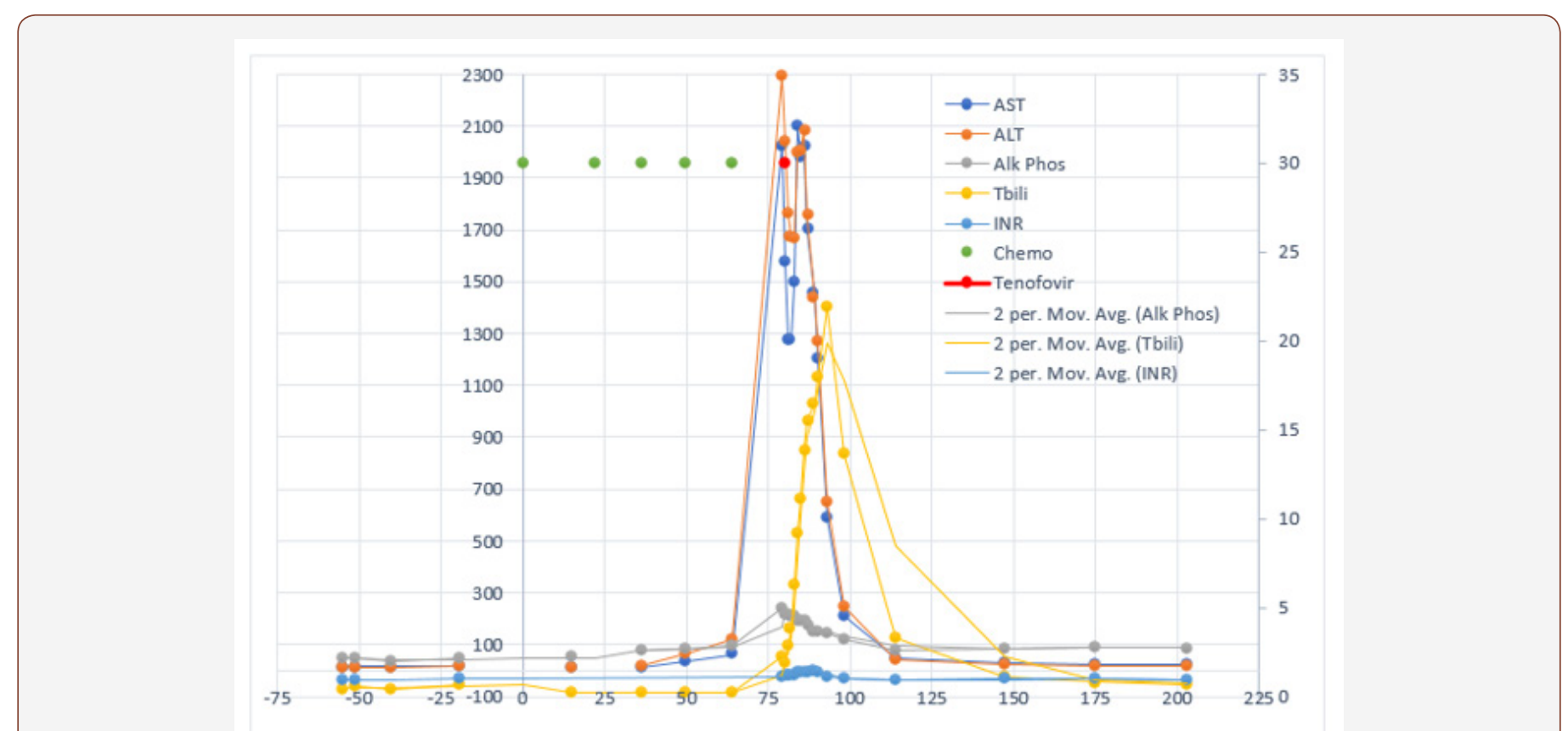

Figure 1: Liver function tests before, during and after HBV reactivation.

\section{Discussion}

It estimated that two billion people worldwide have been infected with HBV worldwide. Additionally, there are approximately 250-300 million asymptomatic chronic HBV carriers worldwide and 862,000 to 2.2 million people living with HBV in the United States, the latter including immigrants from endemic countries [1-5]. Due to the persistence of latent HBV in hepatocytes, HBV reactivation may occur after exposure to chemotherapy induced immunosuppression. While the risk of HBV reactivation for treatment of hematologic malignancies is well established, there is a paucity of literature in solid tumors. Estimates of the risk of reactivation in asymptomatic HBV carriers with solid tumors range from $3-50 \%$ depending on tumor type, chemotherapy to be administered, and HBsAg status. It is more common in patients with positive HBsAg than negative HBsAg and positive HBcAb; reported as $20 \%$ to $50 \%$ and 3 to $45 \%$, respectively [5-20]. HBV reactivation is defined as an increase in HBV DNA by at least 10fold in those with previously detectable HBV DNA or de novo detection of HBV DNA and HBsAg positivity. [8,21] The natural history of HBV reactivation is characterized by an asymptomatic rapid rise in HBV DNA frequently accompanied by HbsAg and HbeAg positivity, often followed by a reactivation related hepatitis with a rise in serum ALT levels and bilirubin. [22] The onset may occur as early as 2 weeks after chemotherapy initiation and up to a year after cessation of chemotherapy. HBV reactivation often results in asymptomatic self-limited hepatitis and will resolve with the cessation of chemotherapy and initiation of anti-viral therapy but can lead to acute liver failure or persistent liver injury and even death in a subset of patients [6,23]. Some suggested risk factors for HBV reactivation include male sex, older age, presence of cirrhosis, diagnosis of lymphoma, breast cancer, hepatocellular carcinoma (HCC), use of anthracyclines or steroids, high baseline HBV DNA, HBeAg positivity, chronic hepatitis B with positive HBsAg positivity, HBV genotype, and low pre-treatment white blood cell count and number of cycles for transarterial chemoembolization (TACE) in HCC. [6,8,17,18,24-26] Risk of HBV reactivation has been 
stratified into high, moderate and low risk or $\geq 10 \%, 1 \%-10 \%$, and $<1 \%$, respectively based on HBsAg positivity and type of chemotherapy or immunosuppressive therapy predominantly in the gastroenterology literature. [8] Immunosuppressive therapies deemed high risk for reactivation are B-cell depleting agents such as rituximab, high dose corticosteroids, anthracyclines, potent Tumor Necrosis Factor-alpha (TNF- $\alpha$ ) inhibitors, and local therapy for HCC including TACE. [9,12-15,27-31] Therapies that have been associated with moderate risk are other systemic chemotherapies (e.g. platinum), less potent TNF- $\alpha$ inhibitors, cytokine-based therapies, calcineurin inhibitors, tyrosine kinase inhibitors, proteasome inhibitors, histone deacetylase inhibitors, and moderate dose corticosteroids. Whereas, moderate and low dose prednisone and methotrexate are associated with low risk of reactivation [8].

The US Centers for Disease Control and Prevention(CDC), European Society for Medical Oncology (ESMO), European Association for the Study of the Liver (EASL), and American Association for the Study of Liver Disease (AALD) recommend universal screening of patients for hepatitis B prior to initiating cytotoxic chemotherapy.[32-40] Whereas, the American Society of
Clinical Oncology (ASCO), National Comprehensive Cancer Network (NCCN), and American Gastroenterological Association Institute (AGA) recommend risk based screening. [37,41] Patients undergoing screening should be tested for HBsAg, surface antibody (HBsAb), and HBcAb with HBV DNA and HBeAg testing if either HBsAg or $\mathrm{HBcAb}$ are positive. About $2-41 \%$ of patients with isolated positive HBcAb have positive HBV DNA, or occult hepatitis B infection. [42] Figure 2 displays a proposed algorithm for hepatitis B screening for patients receiving chemotherapy for solid tumors based on available guidelines. Inconsistent guidelines may contribute to low rates of HBV screening often less than $20 \%$ for patients undergoing chemotherapy. $[1,43,44]$ Low screening rates have also been attributed to underestimation of the risk of $\mathrm{HBV}$ reactivation, perception that there is inadequate evidence to support universal screening and of low rates of HBV infection. [45,46] There are concerns that universal screening may lead to unnecessary costs and over-treatment. Studies of the cost-effectiveness of universal HBV screening in patients with solid tumors undergoing cytotoxic chemotherapy have been conflicting. [47-49] However, risk-based approaches may be more time consuming and require oncologist knowledge of HBV reactivation risk factors and chemotherapy agents associated with increased risk (Figure 2).

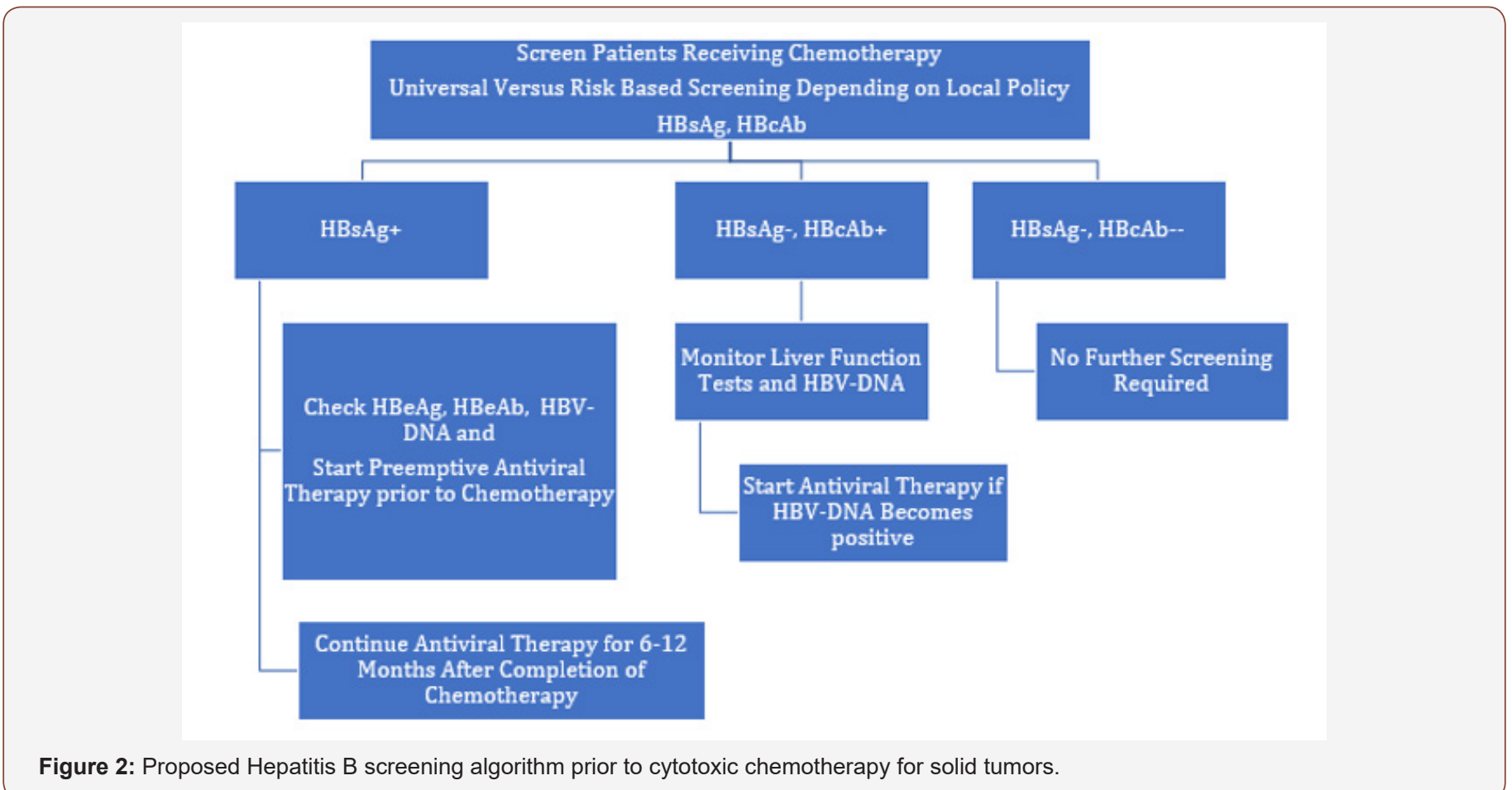

Preemptive antiviral therapy significantly reduces HBV reactivation, hepatic failure, mortality and chemotherapy disruption. $[1,5,12,15,17,29,32,50-56]$ Therefore, it is recommended that HBsAg positive patients receive preemptive treatment with nucleoside reverse transcription inhibitors. Antivirals should be initiated prior to chemotherapy administration and continued for 6 to 12 months after completion of chemotherapy. Further studies are necessary to guide the optimal duration of antiviral prophylaxis after completion of chemotherapy. While lamivudine has historically been used for most trials of HBV reactivation prophylaxis, potent $\mathrm{HBV}$ antivirals with a high barrier to resistance, such as entecavir or tenofovir, are generally first line. Moreover, a randomized controlled trial of lamivudine versus entecavir prophylaxis for patients receiving chemotherapy for lymphoma revealed significantly lower rates of HBV reaction, HBV related hepatitis, and chemotherapy disruption in the entecavir group. [57] For HBsAg-negative/HbcAb-positive patients, on-demand antiviral therapy is recommended. The most recent ASCO provisional clinical opinion update suggests monitoring HBV DNA and ALT every 3 months for patients with HBsAg-negative/HBcAB-positive patients while receiving chemotherapy and initiating antiviral therapy if HBV reactivation occurs. [37] It is important to work with 
local laboratories to ensure that sensitive diagnostic methods for HBV DNA monitoring are utilized. However, there is no standard monitoring practices and limited data available to guide practice.

\section{Conclusion}

HBV reactivation is a potentially serious, even life-threatening complication of immunosuppressive chemotherapy for solid tumors in patients with history of HBV infection or occult hepatitis $B$ infections. As this complication can be prevented and effectively managed with antiviral therapy, screening and monitoring patients imperative. More randomized controlled trials are required to help guide safe and cost-effective screening practices and duration of antiviral therapy. Additionally, quality improvement initiatives are needed to optimize screening practices for patients at high risk.

\section{Acknowledgement}

All authors contributed to manuscript writing and editing.

\section{Conflict of Interest}

Dr. Algaze and Dr. Donovan have no conflicts of interest to report. Dr. Kang has accepted speaker and consulting fees from Puma Biotechnology and consulting fees from Bristol Myers Squibb.

\section{References}

1. Wang Y, Luo X-M, Yang D, Zhang J, Zhuo H-Y, et al. (2013) Testing for hepatitis $B$ infection in prospective chemotherapy patients: a retrospective study. World Journal of Gastroenterology: WJG 19(6): 923930.

2. Organization WH (2017) Global hepatitis report 2017: World Health Organization.

3. Roberts H, Kruszon-Moran D, Ly KN, Hughes E, Iqbal K, et al. 2016 Prevalence of chronic hepatitis B virus (HBV) infection in US households: National Health and Nutrition Examination Survey (NHANES), 1988-2012. Hepatology 63(2): 388-397.

4. Patel EU, Thio CL, Boon D, Thomas DL, Tobian AA (2019) Prevalence of hepatitis B and hepatitis D virus infections in the United States, 20112016. Clinical Infectious Diseases.

5. Long M, Jia W, Li S, Jin L, Wu J, et al. (2011) A single-center, prospective and randomized controlled study: Can the prophylactic use of lamivudine prevent hepatitis B virus reactivation in hepatitis B s-antigen seropositive breast cancer patients during chemotherapy? Breast cancer research and treatment 127(3): 705 .

6. Yeo W, Chan PK, Zhong S, Ho WM, Steinberg JL, et al. (2000) Frequency of hepatitis $B$ virus reactivation in cancer patients undergoing cytotoxic chemotherapy: a prospective study of 626 patients with identification of risk factors. Journal of medical virology. 62(3): 299-307.

7. Yeo W, Chan PK, Hui P, Ho WM, Lam KC, et al. (2003) Hepatitis B virus reactivation in breast cancer patients receiving cytotoxic chemotherapy: a prospective study. Journal of medical virology. 70(4):553-561.

8. Loomba R, Liang TJ (2017) Hepatitis B reactivation associated with immune suppressive and biological modifier therapies: current concepts, management strategies, and future directions. Gastroenterology. 152(6):1297-1309.

9. Kim MK, Ahn JH, Kim S-B, Im Y-S, Im Lee S, et al. (2007) Hepatitis B reactivation during adjuvant anthracycline-based chemotherapy in patients with breast cancer: a single institution's experience. The Korean journal of internal medicine 22(4):237.

10. Ide Y, Ito Y, Takahashi S, Tokudome N, Kobayashi K, et al. (2013) Hepatitis $B$ virus reactivation in adjuvant chemotherapy for breast cancer. Breast Cancer 20(4): 367-370.
11. Hicks LK, Feld JJ, Saluja R, Truong J, Haynes AE, et al. (2016) Hepatitis $B$ reactivation in patients with solid tumors: A systematic review and meta-analysis. American Society of Clinical Oncology.

12. Dai M, Wu P, Shyu R, Lu J, Chao TY (2004) Hepatitis B virus reactivation in breast cancer patients undergoing cytotoxic chemotherapy and the role of preemptive lamivudine administration. Liver international 24(6): 540-546.

13. Ling W, Soe P, Pang A, Lee S (2013) Hepatitis B virus reactivation risk varies with different chemotherapy regimens commonly used in solid tumours. British journal of cancer 108(10): 1931.

14. Zhang S-S, Liu J-X, Zhu J, Xiao M-B, Lu C-H, et al. (2019) Effects of TACE and preventive antiviral therapy on HBV reactivation and subsequent hepatitis in hepatocellular carcinoma: a meta-analysis. Japanese journal of clinical oncology.

15. Jang JW, Choi JY, Bae SH, Yoon SK, Chang UI, et al. (2006) A randomized controlled study of preemptive lamivudine in patients receiving transarterial chemo-lipiodolization. Hepatology 43(2): 233-240.

16. Yeo W, Lam K, Zee B, Chan P, Mo F, et al. (2004) Hepatitis B reactivation in patients with hepatocellular carcinoma undergoing systemic chemotherapy. Annals of oncology 15(11): 1661-1666.

17. Lao XM, Luo G, Ye LT, Luo C, Shi M, et al. (2013) Effects of antiviral therapy on hepatitis B virus reactivation and liver function after resection or chemoembolization for hepatocellular carcinoma. Liver International 33(4): 595-604.

18. Peng JW, Lin GN, Xiao JJ, Jiang XM (2012) Hepatitis B virus reactivation in hepatocellular carcinoma patients undergoing transcatheter arterial chemoembolization therapy. Asia-Pacific Journal of Clinical Oncology 8(4): 356-361.

19. Cheng JCH, Liu MC, Tsai SY, Fang WT, Jer-Min Jian J, et al. (2004) Unexpectedly frequent hepatitis B reactivation by chemoradiation in postgastrectomy patients. Cancer 101(9): 2126-2133.

20. Hagiwara S, Sakurai T, Nishina S, Tanaka K, Ikeda M, et al. (2012) Characteristic pattern of reactivation of hepatitis B virus during chemotherapy for solid cancers. Digestive Diseases 30(6): 541-546.

21. Hoofnagle JH (2009) Reactivation of hepatitis B. Hepatology 49(S5): S156-S165.

22. Yeo W, Chan PK, Chan HL, Mo FK, Johnson PJ (2001) Hepatitis B virus reactivation during cytotoxic chemotherapy-enhanced viral replication precedes overt hepatitis. Journal of medical virology 65(3): 473-477.

23. Steinberg JL, Yeo W, Zhong S, Chan JY, Tam JS, et al. (2000) Hepatitis B virus reactivation in patients undergoing cytotoxic chemotherapy for solid tumours: precore/core mutations may play an important role. Journal of medical virology 60(3): 249-255.

24. Yeo W, Zee B, Zhong S, Chan P, Wong W, et al. (2004) Comprehensive analysis of risk factors associating with Hepatitis B virus (HBV) reactivation in cancer patients undergoing cytotoxic chemotherapy. British journal of cancer 90(7): 1306-1311.

25. Shouval D, Shibolet $O$ (2013) Immunosuppression and HBV reactivation. Seminars in liver disease: Thieme Medical Publishers.

26. Lin CI, Lin ZY, Hsieh MY, Huang CF, Chen SH, et al. (2011) Potential risk factors for the reactivation of the replication of hepatitis $B$ and $C$ viruses after transcatheter arterial chemoembolization of hepatocellular carcinoma. The Kaohsiung journal of medical sciences 27(12): 554-559.

27. Evens A, Jovanovic B, Su Y-C, Raisch D, Ganger D, et al. (2010) Rituximabassociated hepatitis $\mathrm{B}$ virus (HBV) reactivation in lymphoproliferative diseases: meta-analysis and examination of FDA safety reports. Annals of Oncology 22(5): 1170-1180.

28. Mozessohn L, Chan K, Feld J, Hicks L (2015) Hepatitis B reactivation in HBsAg-negative/HBcAb-positive patients receiving rituximab for lymphoma: a meta-analysis. Journal of viral hepatitis 22(10): 842-849.

29. Paul S, Saxena A, Terrin N, Viveiros K, Balk EM, et al. (2016) Hepatitis $\mathrm{B}$ virus reactivation and prophylaxis during solid tumor chemotherapy: a systematic review and meta-analysis. Annals of internal medicine 164(1): 30-40. 
30. Hsu C-H, Hsu H-C, Chen H-L, Gao M, Yeh P-Y, et al. (2004) Doxorubicin activates hepatitis $B$ virus (HBV) replication in HBV-harboring hepatoblastoma cells. A possible novel mechanism of HBV reactivation in HBV carriers receiving systemic chemotherapy. Anticancer research 24(5A): 3035-3040.

31. Jun BG, Kim YD, Kim SG, Kim YS, Jeong SW, et al. (2018) Hepatitis B virus reactivation after radiotherapy for hepatocellular carcinoma and efficacy of antiviral treatment: A multicenter study. PloS one 13(7): e0201316.

32. Perrillo RP, Gish R, Falck-Ytter YT (2015) American Gastroenterological Association Institute technical review on prevention and treatment of hepatitis B virus reactivation during immunosuppressive drug therapy. Gastroenterology 148(1): 221-244

33. Liver EAFTSOT (2012) EASL clinical practice guidelines: management of chronic hepatitis B virus infection. Journal of hepatology 57(1): 167185.

34. Weinbaum CM, Mast EE, Ward JW (2009) Recommendations for identification and public health management of persons with chronic hepatitis B virus infection. Hepatology 49(S5): S35-S44.

35. Terrault NA, Lok AS, McMahon BJ, Chang KM, Hwang JP, et al. (2018) Update on prevention, diagnosis, and treatment of chronic hepatitis B: AASLD 2018 hepatitis B guidance. Hepatology 67(4):1560-1599.

36. Taplitz RA, Kennedy EB, Bow EJ, Crews J, Gleason C, et al. (2018) Antimicrobial prophylaxis for adult patients with cancer-related immunosuppression: ASCO and IDSA clinical practice guideline update. Journal of clinical oncology : official journal of the American Society of Clinical Oncology 36(30): 3043-3054.

37. Hwang JP, Somerfield MR, Alston-Johnson DE, Cryer DR, Feld JJ, et al. (2015) Hepatitis B virus screening for patients with cancer before therapy: American Society of Clinical Oncology provisional clinical opinion update. Journal of Clinical Oncology 33(19): 2212.

38. Baden LR, Bensinger W, Angarone M, Casper C, Dubberke ER, et al. (2012) Prevention and treatment of cancer-related infections. Journal of the National Comprehensive Cancer Network 10(11): 1412-1445.

39. Liver EAFTSOT (2017) EASL 2017 Clinical Practice Guidelines on the management of hepatitis B virus infection. Journal of hepatology 67(2): 370-398.

40. De la Revilla J, Calleja J, Abreu L (2013) Cancer treatment in patients with hepatitis. ESMO Handbook: Cancer Treatment in Special Clinical Situations.

41. Reddy KR, Beavers KL, Hammond SP, Lim JK, Falck-Ytter YT (2015) American Gastroenterological Association Institute guideline on the prevention and treatment of hepatitis $B$ virus reactivation during immunosuppressive drug therapy. Gastroenterology 148(1): 215-219.

42. Wu T, Kwok RM, Tran TT (2017) Isolated anti-HBc: the relevance of hepatitis B core antibody-a review of new issues. The American journal of gastroenterology 112(12): 1780

43. Hwang JP, Fisch MJ, Zhang H, Kallen MA, Routbort MJ, et al. (2012) Low rates of hepatitis $\mathrm{B}$ virus screening at the onset of chemotherapy. Journal of oncology practice 8(4): e32-e9.
44. Lee R, Vu K, Bell C, Hicks L (2010) Screening for hepatitis B surface antigen before chemotherapy: current practice and opportunities for improvement. Current Oncology 17(6): 32-38.

45. Lee RS, Bell CM, Singh JM, Hicks LK (2012) Hepatitis B screening before chemotherapy: a survey of practitioners' knowledge, beliefs, and screening practices. Journal of oncology practice 8(6): 325-328.

46. Day FL, Link E, Thursky K, Rischin D (2011) Current hepatitis B screening practices and clinical experience of reactivation in patients undergoing chemotherapy for solid tumors: a nationwide survey of medical oncologists. Journal of oncology practice 7(3): 141-147.

47. Day FL, Karnon J, Rischin D (2011) Cost-effectiveness of universal hepatitis B virus screening in patients beginning chemotherapy for solid tumors. Journal of Clinical Oncology 29(24): 3270-3277.

48. Tan G, Zhou K, Tan CH, Matchar DB, Farid M, et al. (2016) Cost effectiveness of universal hepatitis $\mathrm{B}$ virus screening in patients beginning chemotherapy for sarcomas or GI stromal tumors. Journal of global oncology 2(4): 186-199.

49. Konijeti GG, Grandhe S, Konerman M, Lane J, Shrime M, et al. (2019) Cost-Effectiveness Analysis of Screening for Hepatitis B Virus Infection in Patients with Solid Tumors before Initiating Chemotherapy. Clinical Gastroenterology and Hepatology.

50. Lau GK, Yiu HH, Fong DY, Cheng H-C, Au W-Y, et al. (2003) Early is superior to deferred preemptive lamivudine therapy for hepatitis B patients undergoing chemotherapy. Gastroenterology 125(6): 17421749.

51. Lee HJ, Kim DY, Keam B, Lee JH, Han S-W, et al. (2014) Lamivudine prophylaxis for hepatitis B virus carrier patients with breast cancer during adjuvant chemotherapy. Breast cancer 21(4): 387-393.

52. Loomba R, Rowley A, Wesley R, Liang TJ, Hoofnagle JH, et al. (2008) Systematic review: the effect of preventive lamivudine on hepatitis B reactivation during chemotherapy. Annals of Internal Medicine 148(7): 519-528.

53. Liu J-Y, Sheng Y-J, Ding X-C, Tang H, Tong S-W, et al. (2015) The efficacy of lamivudine prophylaxis against hepatitis $\mathrm{B}$ reactivation in breast cancer patients undergoing chemotherapy: a meta-analysis. Journal of the Formosan Medical Association 114(2): 164-173.

54. Yeo W, Ho WM, Hui P, Chan PK, Lam KC, et al. (2004) Use of lamivudine to prevent hepatitis $\mathrm{B}$ virus reactivation during chemotherapy in breast cancer patients. Breast cancer research and treatment 88(3): 209-215.

55. Yeo W, Hui EP, Chan AT, Ho WM, Lam KC, et al. (2005) Prevention of hepatitis $B$ virus reactivation in patients with nasopharyngeal carcinoma with lamivudine. American journal of clinical oncology 28(4): 379-384.

56. Sohn BS, Ahn JH, Jung KH, Ahn S-H, Son BH, et al. (2011) Updated longitudinal data on acute exacerbation of chronic hepatitis B in patients with breast cancer receiving anthracycline-based adjuvant chemotherapy: therapeutic vs. pre-emptive use of lamivudine. Japanese journal of clinical oncology 41(9): 1059-1066.

57. Huang H, Li X, Zhu J, Ye S, Zhang H, et al. (2014) Entecavir vs lamivudine for prevention of hepatitis $B$ virus reactivation among patients with untreated diffuse large B-cell lymphoma receiving R-CHOP chemotherapy: a randomized clinical trial. Jama 312(23): 2521-2530. 